\title{
Why choose 3D bioprinting? Part II: methods and bioprinters
}

\author{
Yong $\mathrm{He}^{1} \cdot$ Zeming $\mathrm{Gu}^{1} \cdot$ Mingjun $\mathrm{Xie}^{1} \cdot$ Jianzhong $\mathrm{Fu}^{1} \cdot \mathrm{Hui} \mathrm{Lin}^{2}$
}

Published online: 1 February 2020

(c) Zhejiang University Press 2020

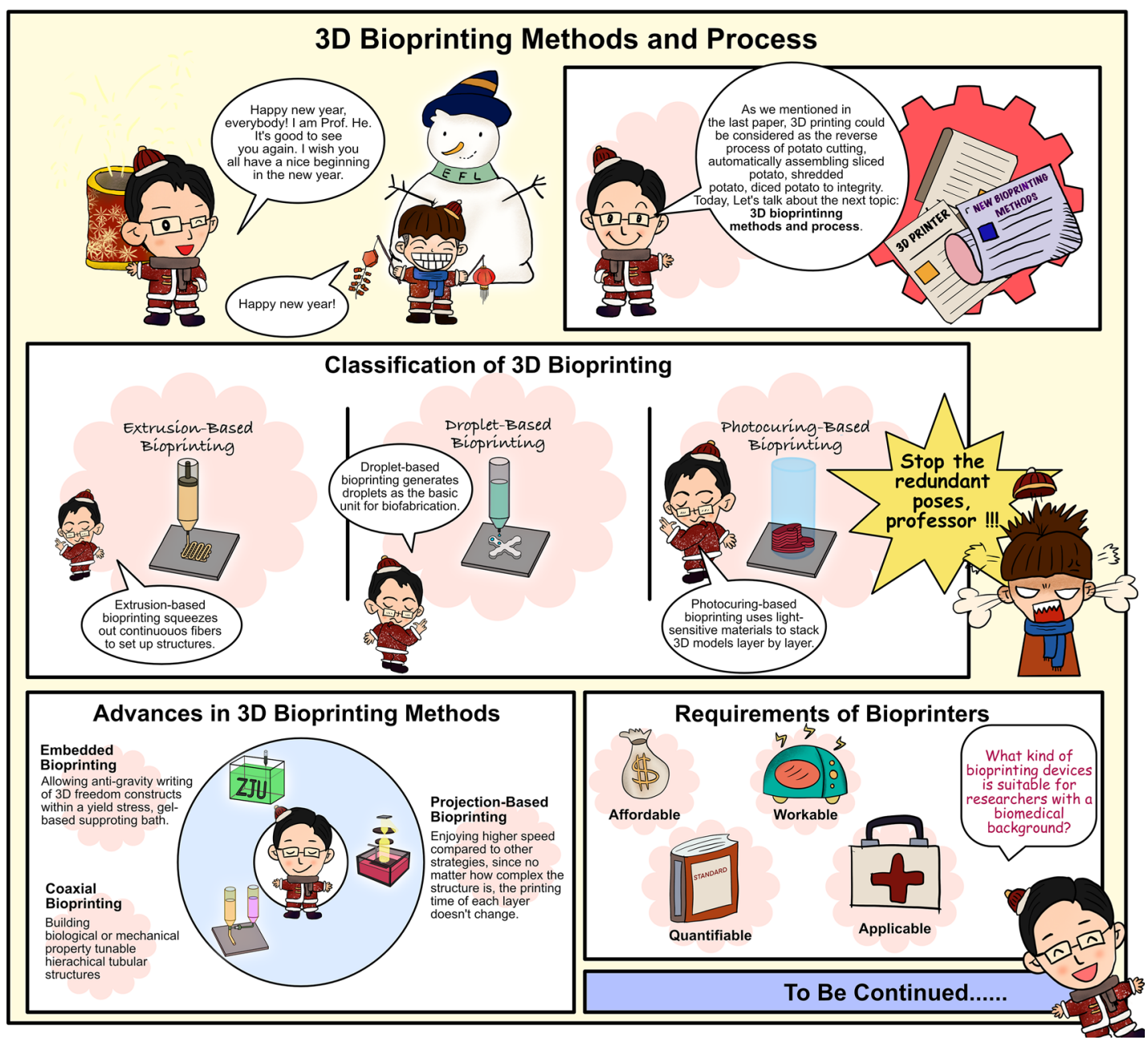

Yong $\mathrm{He}$

yongqin@zju.edu.cn

\section{Classification of 3D bioprinting}

1 State Key Laboratory of Fluid Power and Mechatronic Systems, School of Mechanical Engineering, Zhejiang University, Hangzhou 310027, China

2 Department of General Surgery, Sir Run Run Shaw Hospital, School of Medicine, Zhejiang University, Hangzhou 310058, China

As we mentioned in the last editorial, 3D printing, also known as additive manufacturing, could be considered as the reverse process of potato cutting, automatically assembling sliced potato, shredded potato, diced potato to integrity [1]. Generally speaking, cell-laden 3D bioprinting can be 
classified into three types: extrusion-based, droplet-based and photocuring-based bioprinting according to different printing principles. Extrusion-based bioprinting squeezes out continuous hydrogel fibers to set up structures; dropletbased bioprinting generates droplets as the basic unit for biofabrication; and photocuring-based bioprinting utilizes the characteristics of light-sensitive materials, to stack 3D models layer-by-layer. Different bioprinting approaches own diverse characteristics facing various scenarios and have specific requirements for bioinks.

\section{Extrusion-based bioprinting}

Extrusion-based bioprinting is the most widely used bioprinting method at present, which is suitable for a broad range of biocompatible materials. Based on different liquid dispensing approaches, pneumatic-driven, piston-driven and screw-driven extrusion systems have been applied to extrude cell-laden bioinks through a nozzle orifice in manner of continuous filaments. 3D complex structures are obtained after repetition of layer-by-layer stacking of fibers on a receiving substrate.

For extrusion-based bioprinting, scholars often choose bioinks with temperature-sensitive, or shear thinning properties which meet the printability requirements. Materials with viscosities ranging from 30 to $6 \times 10^{7} \mathrm{mPa} / \mathrm{s}$ have been shown to be compatible with extrusion-based bioprinters [2]. It is a common sense that materials with higher viscosities offer more support for maintaining bioprinted structures, while lower viscosities materials provide better micro-environment for cell viability and functionalization. Thereby, the wide range of printable biomaterial viscosities means abundant selection of bioinks facing different scenarios. Furthermore, multi-material bioprinting, which means utilizing two and more kinds of biomaterials to build a structure in collaboration, can also be closely combined with extrusion-based bioprinting. Nevertheless, the resolution of extrusion-based bioprinting is usually about 200 microns, which not only makes it difficult to accurately simulate tissue structures, but also limits its wide applications. Besides, shear force during high-viscosity bioinks extrusion process might result in cell damage.

\section{Droplet-based bioprinting}

Other than extrusion-based bioprinting using fibers as basic unit, droplet-based bioprinting employs discrete droplets to stack into 3D models. On the basis of different droplets forming principles, droplet-based bioprinting can be roughly divided into inkjet bioprinting [3], electrohydrodynamic jetting (EHDJ) [4], and laser-assisted bioprinting (LAB) [5].

Inkjet bioprinting takes advantage of bioinks physical properties, including viscosity, surface tension and density, to deposit microspheres onto a receiving substrate. It can be further classified into continuous inkjet (CIJ) bioprinting and drop-on-demand (DOD) inkjet bioprinting, where DOD inkjet bioprinting, including thermal-and piezoelectricinkjet bioprinting, is more commonly applied in tissue engineering field. Due to its accessibility and capability of biomaterials precise control, inkjet bioprinting possesses several advantages such as low cost and high printing precision. However, this technique has several limitations, including nozzle plugging during cell-laden bioinks jetting and considerable damage impacts on cells in the process of inkjet which influences the subsequent functionalization. In addition, this technique has a limited range of available bioinks, particularly in high concentrations; and its nozzle head is damageable.

EHDJ employs an electric field motivated by high voltage to pull droplets out of the nozzle orifice. Variation of the voltage would certainly influence the size of each droplet, where higher voltage brings smaller droplets [6, 7]. Because it avoids excessive pressure during extrusion process of bioink through nozzle, which would affect cell viability, EHDJ is suitable for bioprinting bioinks with high weight/volume ratio and high cell concentration. This bioprinting method has advantages such as low cost, high efficiency, high precision, deposition position controllability and low cell damage, but it also has limitations such as electric accumulation affecting deformation of complex constructions; electric field intensity is related to the height of printed structure, leading to complicated process control and difficult to stack many layers [8].

LAB is a non-contact, nozzle-free bioprinting approach, to precisely deposit bioink droplets. LAB technique contains laser-guidance direct writing (LGDW) and laser-induced forward transfer (LIFT), where LGDW exploits optical trap to guild cells onto a substrate, while LIFT utilizes a focused pulsed laser-induced local evaporation of bioink-coated layer to propel biomaterial toward receiving layer. LAB innately avoids nozzle clogging problems and shows reproducibility because its nozzle-free characteristic, and it can reach higher printing resolution compared to inkjet bioprinting because of its smaller droplet generation. Whereas, several drawbacks still limit the use of LAB technology. For example, the uniformity of the coating layer is not easy to control, manually replacing coating is inefficient, process adjust and control is far too tedious, unable to fabricate complex structures, lack of commercialized devices, etc.

\section{Photocuring-based bioprinting}

Photocuring-based bioprinting solidifies photosensitive polymers to form constructs under precisely controlled lighting with high printing precision and fast printing speed. It can be further classified into stereolithography (SLA), and 
projection-based printing (also called digital light processing, DLP) on account of different light scanning modes.

For bioprinting each layer using SLA technique, laser scans a 2D pattern by passing through its path point-bypoint. When one layer is cured, platform would lower a floor height for the next until the whole structure obtained. Differs from SLA, DLP photo-crosslinking one complete layer instantaneously instead of point-by-point. The printing process can be categorized into bottom-up, or top-down. Using a digital micromirror device (DMD) as dynamic mask, a DLP bioprinter can shift 2D pattern for each layer, which leads to high printing speed. Photocuring-based bioprinting has advantages including nozzle-free and high resolution, while it has several deficiencies such as its non-ideal density and uniformity of loaded cells, ultraviolet exposure and toxic photoinitiators might affect cells activity, and there are not many compatible biomaterials.

\section{Advances in 3D bioprinting methods}

Klebe first demonstrated printing biologics in 1988 [9], and bioprinting has been carried forward since Boland et al. put forward the concept of cell and organ printing in 2003 [10, 11]. Since then, there have been a great many bioprinting methods applied with the rapid technological evolution, and some outdated printing methods have been gradually eliminated, while novel ideas are continuously applied in this field. We believe coaxial bioprinting, DLP and embedded bioprinting, which have approved considerable achievements and showed great potentials, will play increasingly important roles in 3D bioprinting.

\section{Coaxial bioprinting}

Coaxial bioprinting has been a more and more popular extrusion-based bioprinting method since it was introduced to tissue engineering field in 2015 [12], especially in the area of blood vessel biofabrication/vascularization. The greatest advantage of coaxial bioprinting is its capability of building biological/mechanical property tunable hierarchical tubular structures. As is known to all, hydrogels with excellent biocompatibility usually possess insufficient mechanical strength. And it can be solved to a certain extent with core-shell structures by coaxial bioprinting: core material ensures biocompatibility, while shell material provides mechanical strength, or vice versa. Using sacrificial material as the core material would also contribute to conveniently bioprinting hollow tubular structures. In the urgent demand of bioprinted organoids requiring considerable vascularization to ensure the viability and functionality of cells, coaxial bioprinting will play an increasingly important role in in vitro model construction.

\section{Projection-based bioprinting}

As we mentioned above, projection-based printing applies photosensitive biomaterials, gelatin methacryloyl (GelMA) hydrogel for instance, to layer-by-layer stack complex 3D structures under the exposure of particular wavelength of light. In principle, DLP enjoys an exceptional advantage in printing speed compared to other bioprinting strategies, since no matter how complex the structure is, and the printing time of each layer does not change. Besides, compared to traditional extrusion-based, or inkjet bioprinting, DLP owns a much better printing resolution, reproductivity and can fabricate constructs much smoother [13], which certainly leads to an improved bioprinted constructs standardization, structural integrity, mechanical property. There are also no worries about nozzle plugging, or shear stress affecting cell viability. We expect that DLP might replace extrusion-based bioprinting as the most mainstream bioprinting method in the near future.

\section{Embedded bioprinting}

One of the major limitations of traditional bioprinting approaches is that fabrication of discrete patterns, which are not mechanically supported, cannot be achieved. In addition, building structures with bifurcated blood vessels has never been a simple task. To address these problems, embedded bioprinting allows anti-gravity writing of 3D freeform constructs within a yield stress, gel-based supporting bath, which would be further removed post-printing to retrieve models with desired shapes or channels [14-16]. What is more, bioprinting components of human heart at various scales, from capillaries to the full organ, were achieved using embedded bioprinting technology this year [17]. Although it has a relatively narrow selection for bioinks and support materials, embedded bioprinting is still a technology with broad prospects.

\section{Requirements of bioprinters}

With the rapid development of 3D bioprinting in the field of tissue engineering and regenerative medicine, including clinical transplantation, pharmaceutics, high-throughput screening, cancer research and even cosmetology, the demand for bioprinters has risen substantially. A variety of bioprinters have been developed at research institutions/universities all over the world, and numerous companies have sprung up to commercialize advanced bioprinter technologies.

There are a wide variety of bioprinters available in the market, which are usually enormous, multi-functional, expensive and difficult to evaluate. Whereas, what kind of bioprinting devices is suitable for researchers with a biomedical 
background? We believe the future trends of bioprinters are affordable, workable, quantifiable and applicable, which are highly consistent with the demands of more and more scholars entering the field of 3D bioprinting. 3D bioprinting, which is still in the process of gaining popularity, will be a main method to construct $3 \mathrm{D}$ cell culture micro-environment and become a universal approach in biomedical research area.

\section{- Affordable}

With the rapid development of 3D bioprinting, more and more researchers and related industry scholars set foot in this area. 3D bioprinting devices with unreasonable prices would be a barrier for new comers.

- Workable

Highly integrated bioprinters are usually costly and hard to get started for beginners. Workable of bioprinting devices stands for configuration modularized, as easy to use as a kitchen device at the same time. Modularization ensures straightforward, relatively small in size, users can select different modules according to their needs, while compact of bioprinters is to satisfy the requirements for cell printing within a standard clean bench.

\section{- Quantifiable}

The standardization and consistency evaluation of printing process are becoming more and more urgent with the rapid evolution of bioprinting technologies. And a quantitative standard of printing quality should be established both at the level of structure precision and cell growth.

- Applicable

Various cell injuries such as mechanical damage in printing process and cell damage during digestion and metastasis should be taken into consideration to measure the availability of its follow-up clinical application.

\section{Discussion}

3D bioprinting has evolved rapidly over the last three decades, recent studies have made great achievements in organoids bioprinting and in vitro model construction, and a myriad of bioprinting methods have been developed for multiple application scenarios. Nevertheless, 3D bioprinting techniques still have a lot of room for progress, for example, biofabrication resolution, bioprinting speed, applicability to a wide range of bioinks. And multi-scale, multi-material, multi-cell bioprinting are becoming the focus of future development. We believe that affordable, workable, quantifiable and applicable are the future trends of bioprinting devices development. It is expected that 3D bioprinting will continue to evolve to achieve the process from structural similarity to functional realization.

\section{Compliance with ethical standards}

Conflict of interest The authors declare that they have no conflict of interest.

Human and animal rights This article does not contain any studies with human or animal subjects performed by any of the authors.

\section{References}

1. He Y, Xie M, Gao Q, Fu J (2019) Why choose 3D bioprinting? Part I: a brief introduction of $3 \mathrm{D}$ bioprinting for the beginners. Bio-Des Manuf 2(4):221-224

2. Jones N (2012) Science in three dimensions: the print revolution. Nature 487(7405):22-23

3. Iwanaga S, Arai K, Nakamura M (2015) Inkjet bioprinting essentials of 3D biofabrication and translation. Elsevier, Amsterdam, pp 61-79

4. Huang Y, Bu N, Duan Y, Pan Y, Liu H, Yin Z, Xiong Y (2013) Electrohydrodynamic direct-writing. Nanoscale 5(24):12007-12017

5. Guillotin B, Ali M, Ducom A, Catros S, Keriquel V, Souquet A, Remy M, Fricain J-C, Guillemot F (2013) Laser-assisted bioprinting for tissue engineering biofabrication. Elsevier, Amsterdam, pp $95-118$

6. Workman VL, Tezera LB, Elkington PT, Jayasinghe SN (2014) Controlled generation of microspheres incorporating extracellular matrix fibrils for three-dimensional cell culture. Adv Funct Mater 24(18):2648-2657

7. Gasperini L, Maniglio D, Migliaresi C (2013) Microencapsulation of cells in alginate through an electrohydrodynamic process. J Bioact Compat Polym 28(5):413-425

8. Xie M, Gao Q, Zhao H, Nie J, Fu Z, Wang H, Chen L, Shao L, $\mathrm{Fu}$ J, Chen Z, He Y (2019) Electro-assisted bioprinting of lowconcentration GelMA microdroplets. Small 15(4):e1804216

9. Klebe RJ (1988) Cytoscribing: a method for micropositioning cells and the construction of two-and three-dimensional synthetic tissues. Exp Cell Res 179(2):362-373

10. Mironov V, Boland T, Trusk T, Forgacs G, Markwald RR (2003) Organ printing: computer-aided jet-based 3D tissue engineering. Trends Biotechnol 21(4):157-161

11. Wilson WC Jr, Boland T (2003) Cell and organ printing 1: protein and cell printers. Anat Rec Part A 272(2):491-496

12. Gao Q, He Y, Fu JZ, Liu A, Ma L (2015) Coaxial nozzle-assisted $3 \mathrm{D}$ bioprinting with built-in microchannels for nutrients delivery. Biomaterials 61:203-215

13. Pyo SH, Wang P, Hwang HH, Zhu W, Warner J, Chen S (2017) Continuous optical 3D printing of green aliphatic polyurethanes. ACS Appl Mater Interfaces 9(1):836-844

14. Highley CB, Rodell CB, Burdick JA (2015) Direct 3D printing of shear-thinning hydrogels into self-healing hydrogels. Adv Mater 27(34):5075-5079

15. Hinton TJ, Jallerat Q, Palchesko RN, Park JH, Grodzicki MS, Shue HJ, Ramadan MH, Hudson AR, Feinberg AW (2015) Three-dimensional printing of complex biological structures by freeform reversible embedding of suspended hydrogels. Sci Adv 1(9):e1500758

16. Bhattacharjee T, Zehnder SM, Rowe KG, Jain S, Nixon RM, Sawyer WG, Angelini TE (2015) Writing in the granular gel medium. Sci Adv 1(8):e1500655

17. Lee A, Hudson A, Shiwarski D, Tashman J, Hinton T, Yerneni S, Bliley J, Campbell P, Feinberg A (2019) 3D bioprinting of collagen to rebuild components of the human heart. Science 365(6452):482-487 